\title{
Removal of the Bad Effects of D-Galactosamine on Blood Lipids of Male Wistar Rats By Black and Green Tea Consumption
}

\author{
Madeha N. Al-seeni*, Abeer A. Banjabi and Karima S. Mohamed \\ Department of Biochemistry, Faculty of Science, King Abdulaziz University, PO. Box 42805, Jeddah 21551, \\ Saudi Arabia.*email: nmalsiny@hotmail.com
}

\begin{abstract}
There is an increase in overall health awareness in Saudi Arabia due to a rising rate of diseases such as diabetes and heart diseases in addition to obesity. Thus, Saudi Ministry of Health initiates many programs to improve their eating and drinking habitats. Black and green tea were used as healthy drinks to alternative highcalorie, sugary drinks. The effect of black and green tea consumption on serum lipid and glucose levels in untreated and galactosamine treated male Wistar rats was detected. In this study, male Wistar rats (90) were divided to 3 groups, the first one, 30 rats that drank water, the second group, 30 rats drank $2 \%$ green tea and the third group is 30 rats that drank $2 \%$ black tea for 4 weeks. Each rat group was separated into two subgroups, the first intraperitoneally injected with D-Galactosamine (GalN) and the second group was injected with saline solution. Analysis of blood indicated that serum triglyceride levels (one of the hepatotoxic parameters) showed a marked reduction in response to GalN administration by $29.21 \%, 36.70 \%$ and $26.94 \%$ in control, green tea and black tea groups, respectively, as compared to those untreated animals. Moreover, in the comparison between the groups of treated animals, rats drinking green tea recorded the lowest serum triglyceride level as compared to rats consuming either water or black tea. Black tea consuming groups recorded $56.73 \%$ reduction in serum total lipids as compared to the control group. Regarding serum glucose level, treated rats drinking tea (green or black) showed a significant reduction as compared to treated rats drinking water. Serum total cholesterol levels were significantly elevated in response to GalN injection in rats drinking black tea by $45.22 \%$ as compared to untreated ones, while a small and not significant increase was recorded in green tea group.

Keywords: male rats, lipids, D-Galactosamine, blood, back tea, green tea, liver, cholesterol
\end{abstract}

\section{Introduction}

Plants played a significant role in maintaining human health and improving the quality of human life for thousands of years, and have served humans well as valuable components of seasonings, cosmetics, dyes, medicines, and beverages (Sánchez et al., 2016). On a global level, tea is the most popular beverage and about 3 billion kilograms of tea are produced and consumed yearly (Yang and Landau, 2000). Green tea leaves of Camellia sinensis was consumed mainly in Japan, China and Arab countries. Black tea follows some of the processing steps used for green tea, but with the critical difference that the leaves are bruised, crushed, or broken, thus allowing polyphenol oxidases in the leaf to generate theaflavins, thearubigins and other complex polyphenols from the endogenous catechins. The main constituents of green tea leaves belong to the polyphenol group accounting for 25-35\% on a dry weight basis (Balentine et al., 1997 and Hara et al., 1995). In black tea, the oxidation of polyphenols during processing leads to the formation of catechins and gallic acid complexes such as theaflavins, theaflavinic acids, thearubigins or theasinensis, and of proanthocyanidin polymers (Balentine et al., 1997 and Hara et al.,1995). Since the liver plays a central role in the regulation of lipid metabolism, and with regard to prevention of liver injury, the influence of tea consumption on lipid profile should be investigated. Also, research is highlighting that there may be a beneficial relationship between tea drinking and serum concentration of lipids. Imai \& Nakachi, (1995) indicated an inverse correlation between tea consumption and concentration of serum total cholesterol. Moreover, the report of De Vos \& De Schrijver, (2003) showed a significant reduction of total cholesterol in plasma of rats consuming black tea. These results were in contrast to other studies in which no correlation between tea consumption and plasma cholesterol was found (Klatsky et al., 1985 and Brown et al., 1993). In addition, Lord, (1999) found that although tea cannot lower blood cholesterol or decrease blood pressure, it may improve the health of the circulatory system and reduce the risk of blood clots. Arab et al., (2009) found that consumption of green and black tea is associated with a lower risk of stroke. Methanolic extracts of green and black teas has excellent antibacterial activity on extended spectrum- $\beta$-Lactamases bacteria (Taherpour et al., 2016). Flavonoids, one class of antioxidant found in tea, may prevent blood platelets from clumping and blocking arteries. Although, Green \& Harari (1992) demonstrated that the effect of drinking green tea but not black tea on plasma lipoproteins appears to be characterized by decreasing LDL-C and increasing HDL-C. Princen et al., (1998) indicated that consumption of black tea or green tea had no effect on plasma HDL-C and LDL-C. However, serum lipid concentrations are 
considered as a major risk factor in cardiovascular disease which is the leading cause of death worldwide. With regard to prevention of cardiovascular disease, the association between tea drinking and serum concentrations of lipids and other markers must be confirmed first. The epidemiologic evidence, however, for an association between regular consumption of tea and coronary heart disease is equivocal (Hollman et al., 1999 and Riemersma et al., 2001). Whereas most studies suggested a decrease in the rate of cardiovascular disease outcomes with increasing tea consumption, studies from the United States (Rosenberg et al., 1988 and Rimm et al., 1996) indicated an increased risk with increasing tea consumption. Plant polyphenols are antioxidant and many common flavonoids are several times more potent than Vitamin C or E (Vinson, 1995). Actually, tea is a rich source of flavonoids (Harborne, 1994). Free radicals are unstable molecules that include the hydrogen atom, nitric oxide and molecular oxygen. In an attempt to stabilize, they attack other molecules in the body potentially leading to cell damage and triggering the formation of another free radical resulting in a chain reaction. Some scientists believe that this type of free radical action has been implicated in certain chronic and aging diseases such as cancer, heart disease, stroke, rheumatoid arthritis, cataracts and Alzheimer's disease while antioxidant compounds inhibit many oxidation reactions caused by free radicals thereby preventing or delaying damage to the cells and tissues. This study aimed to determine the effect of the green and black tea on lipid and sugar rates in blood of male Wistar rats with liver injury induced by GalN injection and comparing the effect of green and black. Therefore, several aspects were measured in blood including serum triglycerides, total lipids, and glucose levels.

\section{Material and Methods}

The D-galactosamine hydrochloride (Sigma Chemical Co.), green and black tea (from the local market, Jeddah) and Kits for biochemical tests (from Crescent Diagnostics, KSA) were used in this study.

\section{Animals}

Male Wister rats, 90 rats of 8 weeks old, weighting $189.57 \mathrm{~g}$ were randomly separated to 3 groups and and each group was seprated to 2 sub-groups as described by Banjabi et al. (2014). The groups were control group (C), treated group with $2 \%$ Green tea group (GT) and treated group with $2 \%$ Black tea group (BT). Each group was divided into two subgroups, the first was injected with a saline solution and the other was injected with GalN, $350 \mathrm{mg} / \mathrm{kg}$ body weight (Sugiyama et al., 1999). All rats ate the same basic diet (Banjabi et al. (2014).

Tea solutions were prepared by boiling $20 \mathrm{~g} / \mathrm{l}$ of dry tea in dist. water for $5 \mathrm{~min}$., then each solution was filtered in dark glass and used as feeding bottles for 4 weeks.

\section{Blood samples collection}

Under diethyl ether anesthesia, blood samples were individually obtained from all rats after 22 hours of the injection (Sugiyama et al., 1999) in centrifuge tubes by cardiac puncture and the tubes were centrifuged at $3000 \mathrm{rpm}$ for $20 \mathrm{~min}$. Serum was collected and stored at $-70{ }^{\circ} \mathrm{C}$ until used.

\section{Blood lipids}

The biochemical analysis of cholesterol (CHOL), triglycerides (TG), low-density lipoproteins cholesterol (LDL-C), and high-density lipoproteins cholesterol (HDL-C) were measured by different methods as explained below:

\section{a. Determination of serum total cholesterol}

The determination of serum cholesterol (CHOL) is a major aid in the diagnosis and classification of lipaemias. Cholesterol esterase catalyses hydrolysis of cholesterol esters to cholesterol, which is oxidized by cholesterol oxidase to yield hydrogen peroxide $\left(\mathrm{H}_{2} \mathrm{O}_{2}\right)$. In a coupled reaction catalyzed by peroxidase, quinoneimine dye (red) is formed from $\mathrm{H}_{2} \mathrm{O}_{2}$, 4- aminoantipyrine and phenol. The absorbance of the dye at $546 \mathrm{~nm}$ is propotional to the concentration of cholesterol in the sample (Richmond, 1973, Allain., 1974).

$$
\begin{aligned}
& \text { Cholesteryl esters }+\mathrm{H}_{2} \mathrm{O} \longrightarrow \text { Cholesterol esterase } \longrightarrow \text { Cholesterol }+ \text { fatty acids } \\
& \text { Cholesterol }+\mathrm{O}_{2} \quad \stackrel{\text { Cholesterol oxidase }}{\longrightarrow} \text { Cholesterol-3-one }+\mathrm{H}_{2} \mathrm{O}_{2} \\
& 2 \mathrm{H}_{2} \mathrm{O}_{2}+4 \text { - aminoantipyrine }+ \text { phenol } \stackrel{\text { Peroxidase }}{\longrightarrow} \text { Quinoneimine }+4 \mathrm{H}_{2} \mathrm{O}
\end{aligned}
$$

\section{b. Determination of serum triglycerides}

The enzymatic method for the quantification of triglycerides (TG) includes the hydrolysis of triglycerides to form glycerol, which is achieved by lipase. The glycerol concentration is determined enzymatically coupled with the Trinder reaction using glycerol kinase, glycerol-3-phosphate oxidase and peroxidase. The end product is a quinoneimine dye the concentration of which at $546 \mathrm{~nm}$ is directly proportional to the concentration of triglycerides in the sample (Fossati \& Prencipe, 1982 and Buculo \& David, 1973). 


$$
\begin{aligned}
& \text { Triglycerides }+\mathrm{H}_{2} \mathrm{O} \quad \text { Lipase } \longrightarrow \text { Glycerol }+ \text { Free fatty acid } \\
& \text { Glycerol + ATP } \\
& \underset{\text { Glycerol Kinase }}{\longrightarrow} \text { Glycerol-3-phosphate + ADP } \\
& \text { Glycerol-3-phosphate }+\mathrm{O}_{2} \underset{\text { Geroxidase }}{\stackrel{\text { Glycerol-3-P oxidase }}{\longrightarrow}} \text { Dihydroxyacetone phosphate }+\mathrm{H}_{2} \mathrm{O} \\
& \mathrm{H}_{2} \mathrm{O}_{2}+4 \text { - Aminoantipyrine }+ \text { chlorophenol } \longrightarrow \text { Quinoneimine dye }
\end{aligned}
$$

\section{c. Determination of plasma high density lipoproteins-cholesterol}

Phosphotungstic acid and magnesium ions specially precipitate low and very low density lipoproteins (LDL-C and VLDL). After centrifugation the cholesterol content of the high density lipoproteins (HDL-C) in the supernatant can be determined using Crescent Diagnostics Cholesterol test kit. The intensity of the color was measured at $546 \mathrm{~nm}$ (Burstein et al., 1970 and Grove, 1979).

\section{d. Calculation of plasma low density lipoprotein-cholesterol} formula:

Low density lipoprotein-cholesterol (LDL-C) can be fairly accurately estimated using the Friedwald

LDL-C $=$ Total cholesterol $-\mathrm{HDL}-\mathrm{C}-$ triglyceride $/ 5$

This formula is based on the assumption that VLDL-C is present in a concentration equal to one-fifth of the triglyceride concentration. This assumption is usually valid for triglyceride concentrations of < $400 \mathrm{mg} / \mathrm{dL}$; at higher triglyceride concentrations, inconsistencies in the VLDL triglyceride/ cholesterol ratio occur, and the formula must not be used (Friedwald et al., 1972).

\section{e. Determination of serum total lipids}

For the determination of serum total lipids a colorimetric assay was utilized using sulfo-phosphovanilic mixture (SPV). The assay of total lipids using the SPV reaction involves adding serum to concentrated sulphuric acid and heating, removal of an aliquot of the mixture, and addition of concentrated phosphuric acid plus vanillin. A pink color is formed, which is read spectrophotometrically at $525 \mathrm{~nm}$ (Chabrole \& Charnnat, 1937).

\section{f. Determination of serum glucose.}

The oxidation of glucose is catalysed by glucose oxidase. The resultant hydrogen peroxide $\left(\mathrm{H}_{2} \mathrm{O}_{2}\right)$ is oxidatively coupled with 4-aminophenazone and phenol in the presence of peroxidase to yield a red quinoneimine dye, the concentration of which at $546 \mathrm{~nm}$ is proportional to the concentration of glucose (Trinder, 1969 and Teuscher \& Richterich, 1971).

$$
\begin{aligned}
& \beta \text {-D-Glucose }+\mathrm{H}_{2} \mathrm{O}+\mathrm{O}_{2} \underset{\text { Glucose oxidase }}{\longrightarrow} \text { D-gluconic acid }+\mathrm{H}_{2} \mathrm{O}_{2} \\
& \mathrm{H}_{2} \mathrm{O}_{2}+4 \text {-aminophenazone }+ \text { phenol Peroxidase } \longrightarrow \text { quinoneimine }+4 \mathrm{H}_{2} \mathrm{O}
\end{aligned}
$$

\section{g. Hepatosomatic index (HSI)}

Liver weight was determined and Hepatosomatic index was calculated using the following equation (Sanchez-Muniz et al., 1991):

$$
\text { HSI = liver weight }(\mathrm{g}) \times 100 / \text { body weight }(\mathrm{g}) \text {. }
$$

\section{Statistical analysis}

Mean \pm standard deviations of each value were calculated and One-way ANOVA between experimental groups were applied. In some cases LSD test and independent t-test for paired data were used and differences at $\mathrm{P}<0.05$ were significant.

\section{Results}

In this study, effect of tea drinking green and black tea with or without GalN injection (treated and untreated rats) on blood sugar and lipids were determined. There was no mortality in animals at all, during the experimental period. Table 1 and Figures 1 showed the effects of green tea and black tea consumption lipids and sugar levels in untreated and GalN- treated rats. GalN administration induced a significant reduction in serum triglycerides levels of all experimental groups (Control, green tea or black tea) versus the untreated ones. Our results showed that there were no significant changes in the serum total lipids between the untreated rats and GalN treated rats of either the control or green tea groups, but the black tea group had significantly higher 
total lipids in GalN- treated rats than untreated rats. GalN -treated rats had higher serum glucose levels in the control group when compared with those untreated rats, while there were no significant differences between GalN- treated and untreated rats in either the green and black tea groups.

Table 2 and Figure 2 showed the effects of green tea and black tea consumption on serum total cholesterol, HDL-C, and LDL-C levels in untreated rats and GalN- treated rats. The results indicated that, while in the black tea group, serum total cholesterol was significantly elevated in response to GalN injection, no statistical differences were observed between untreated rats and GalN- treated rats in either the control or green tea groups. GalN administration did not affect either HDL-C or LDL-C levels of all experimental groups. Table 3 and Figure 3 showed the effects of green tea and black tea consumption on liver weight and hepatosomatic index in galactosamine treated and untreated rats. No statistical differences were recorded in either liver weight or hepatosomatic index between all the groups of GalN-treated animals. Liver weight did not differ statistically between rats consuming water, green tea and black tea. The same results were observed for hepatosomatic index.

\section{Discussion}

The beneficial effect of drinking green tea over black tea may be attributed to its higher content of epicatechins, since they remain relatively unchanged compared with the fresh tea leaves, whereas in black tea, they are mostly oxidized during the fermentation process (Graham, 1992). Since catechins have been considered as the major effective component of green tea, many beneficial effects of green tea are attributed to them. They are powerful antioxidants, which are thought to be at least in part responsible for green tea's hepatoprotective activity (Miyagawa et al., 1997, Yand and Koo, 2000). But this effect of green tea is not dependent on its direct antioxidation effects alone. Green tea catechins have been shown to maintain intracellular protein thiol levels. Protein thiol helps maintain the intracellular reduction-oxidation (redox) balance, protein tertiary configuration and therefore cellular function (Miyagawa et al., 1997). Moreover, Hikino et al., (1985) showed tha catechins had a preventive effect on GalN-induced liver injury while He et al., (2001) found that caffeine in tea acting for liver protection.. In contrast, Sugiyama et al., (1999) and Wada et al., (1999) found that the effect of green tea against GalN-induced liver injury was due to flavonoid, glycosides, theanine and soluble dietary fibers, while tea catechins had lower effects. Such contrasting results, concerning the major effective component of green tea, may be due to a number of confounding factors including diet, tea concentration, tea preparation, animal species and the experimental period. With this information, green tea could be used for liver injury treatment protocol; although more human research is needed in this area before a solid recommendation can be made.

Regarding the effect of liver injury on lipid profile, serum triglyceride levels are considered one of the hepatotoxic parameters (Hasegawa et al., 1995). A marked reduction in serum triglyceride level was noted in response to GalN administration by $29.21 \%, 36.70 \%$ and $26.94 \%$ in control, green tea and black tea groups respectively, as compared to those untreated animals (Table 1). This reduction in serum triglyceride level was an additional evidence of liver injury, since decreased triglyceride level is seen in cases of hepatocellular damage (Hasegawa et al., 1995).

Moreover, in the comparison between the groups of treated animals, rats drinking green tea recorded the lowest serum triglyceride level as compared to rats consuming either water or black tea which means that previous drinking of black tea clearly protected against changes in triglyceride levels, caused by GalN injection. On the other hand, our results were unable to note any significant difference in serum triglyceride levels between all untreated groups receiving black tea, green tea or water. This is not compatible with a study of Kono et al., (1992) that showed an association between consumption of green tea and black tea and reduced triglyceride level. The possible explanation of this discrepancy might be related to the different tea concentrations used in both experiments.

In respect to serum total lipids, our results indicated that they were increased significantly in response to GalN injection in the black tea group by $116.98 \%$ compared with the untreated group. While in untreated rats, black tea consuming groups recorded a $56.73 \%$ reduction in serum total lipids as compared to the control group. This result suggests that black tea has a total lipid lowering effect while green tea failed to induce a significant change.

Regarding serum glucose level, treated rats drinking tea (green or black) showed a significant reduction as compared to treated rats drinking water and the same trend was observed in untreated rats. This was also the case in the previous study of Zeyuan et al., (1998), who reported that green and black tea can decrease blood glucose level of aged rats. Moreover, Li et al., (2006) indicated that tea has been credited with controlling blood glucose levels. It seems worthwhile to suggest that either green tea or black tea may affect glucose metabolism in untreated rats or even in GalN-treated ones.

In the same line of our suggestion, Vinson and Zhang 2005 reported that both green tea and black tea significantly inhibited diabetic cataracts and caused significant reductions in the biochemical pathway 
implicated in the development of the pathology in rats.

The possible explanation of the hypoglycemic effect of tea could be related to its polyphenols. Since they inhibit $\alpha$-amylase activity in saliva and reduce the intestinal amylase activity which in turn lowers the hydrolysis of starch to glucose and reduces glucose assimilation (Hara et al., 1995 a).

Moreover, polyphenols can also decrease digestive enzyme activity and reduce the glucose absorption (Zeyuan et al., 1998). Consistent with this, Simizu et al., (1988) have already isolated soluble polysaccharides from an extract of green tea, which had a hypoglycemic effect in Streptozotocin- induced diabetic rats.

High plasma cholesterol has been ranked as one of the greatest risk factors contributing to the prevalence and severity of coronary heart disease. Also, liver diseases could affect the concentration of serum total cholesterol and lipoproteins (Tietz, 1976).

Regarding the comparison between treated and untreated groups, serum total cholesterol levels were significantly elevated in response to GalN injection in rats drinking black tea by $45.22 \%$ as compared to untreated ones, while a small and not significant increase was found in green tea group. This could mean that rats consuming black tea were more sensitive to the GalN administration than those consuming green tea in respect to the serum total cholesterol level.

However, in the group of untreated rats, our results indicated that black tea had significant hypocholesterolemic effect since it lowered serum total cholesterol by $36.16 \%$ comparing to the control animals consuming water. Also, green tea was able to non-significantly reduce the serum total cholesterol of untreated animals comparing to the control group. These findings with untreated rats, suggested that the hypocholesterolemic activity of tea could contribute to the protection against heart disease. Troup et al. (2015) found that there is little therapeutic benefit to using black tea beverage to prevent hypercholesterolemia progression.

The cholesterol lowering effect of tea has been shown in several studies which suggested an inverse correlation between tea consumption and concentration of serum total cholesterol (Imai \& Nakachi, 1995 and Kono et al., 1992). Also, the report of De Vos \& De Schrijver, (2003) showed a significant reduction of total cholesterol (35\%) in plasma of rats consuming black tea. This is in line with our results which indicated that there was a strong tendency of total cholesterol reduction in serum of rats drinking black tea by $36.16 \%$.

However, our results concerning the hypocholesterolemic effect of tea was not in line with other studies (Klatsky et al., 1985 and Brown et al., 1993) showing negative correlation between tea consumption and plasma cholesterol.

Hypocholesterolemic effect of tea may be caused by stimulated intestinal fermentation and formation of acetate by black tea polyphenols in the caecum and the colon (De Vos \& De Schrijver., 2003). Other potential mechanisms may be involved including: the promotion of cholesterol and bile acid excretion (Yang \& Koo., 2000) or the inhibition of cholesterol and bile acid absorption (Chan et al., 1999).

The hypocholesterolemic effect of tea may play an important role in cholesterol metabolism, which can be regulated by the hepatic synthesis of cholesterol and the biliary excretion of sterols and bile acids (Ramesha et al., 1980).

The results obtained in this study failed to show significant differences between the three groups of the GalN-treated rats in either HDL-C or LDL-C levels and these observations suggest that it may be possible that either green tea or black tea was not able to affect HDL-C or LDL-C in liver injury-induced rats, although further evidence is required to confirm this.

On the basis of epidemiological studies, higher HDL-C: LDL-C ratio could be interpreted as an indicator of reduced heart disease (Gordon et al., 1977). Our finding in untreated rats showed that rats drinking black tea had significantly lower LDL-C value as compared with the control group. Moreover, they had a higher HDL-C: LDL-C ratio (111.66\%) as compared to those drinking green tea $(36.67 \%)$. This means that black tea could increase this ratio; therefore, it is effective in reducing the risk of coronary heart disease.

In contrast, Green and Harari (1992) and Imai and Nakachi, (1995) demonstrated that the effect of drinking green tea but not black tea on plasma lipoproteins appears to be characterized by decreasing LDL-C and increasing HDL-C. On the other hand, Princen et al., (1998) indicated that consumption of black or green tea had no effect on plasma HDL-C and LDL-C. These contrasting results may be due to different tea concentrations, different experimental conditions and different methods of preparation used in these studies.

In general, the present study indicated that food intake and body weight of rats drinking tea (green and black) was significantly lower than that of rats drinking water during the experimental period. Moreover, the green tea group consumed less food than the black tea group. In accordance with our results, previous report of Naismith et al., (1969) showed that growth and food intake of rats were suppressed by dietary caffeine containing beverages. Furthermore, He et al., (2001) suggested that the effect of green tea on growth and food intake could be ascribed to caffeine, while Yang \& Landau, (2000) reported that decreased nutrient absorption and increased energy expenditure may both contribute to the suppression of growth and food intake in response to tea consumption. On the other hand, the studies of Greger et al., (1988) and De Vos \& De Schrijver, (2003) 
showed that consumption of black tea did not affect daily food intake nor body weight gain. The authors of the last study mentioned that the tea concentration used was not sufficient to induce a decrease in food intake and body weight gain. Controversially, Miura et al., (2001) found that the body weight in the tea group were greater than those in the control group. However, the data of the present study is not sufficient to evaluate the practical benefit of tea since this experiment employed a single intraperitoneal administration of a lethal dose of GalN, which is different from the actual exposure route and frequency of intake for humans. To clarify the benefits of tea, it is important, in practical terms, to further examine the complete effectiveness of tea after repeated administrations of this toxicant, to assess the influence of different tea fractions and different tea concentrations. In addition, it's also important to evaluate undesirable health- related consequences that may arise from ingestion of large amounts of tea.

\section{References}

[1]. Allain C.C., Poon L.S., Chan C.S., Richmond W. and Fu P.C. (1974) Enzymatic determination of total serum cholesterol. Clin. Chem. 20: 470-475.

[2]. Arab L, Liu W, Elashoff D (2009). Consumption of Green and Black Tea Is Associated With a Lower Risk of Stroke. Neurology Reviews. May; 17(5):7

[3]. Balentine D.A., Wiseman S.A. and Bouwens L.C. (1997) The chemistry of tea flavonoids. Crit. Rev. Food Sci. Nutr. 37: 693-704

[4]. Banjabi A A., Karima S. Mohamed and Madeha N. Al-seeni (2014). Protective effect of green or black tea on D-Galactosamine induced liver injury in male Wistar rats. Life Science J. 11(3):242-249]

[5]. Brown A., Botton-Smith C., Woodward M. and Tunstall-Pedoe H. (1993) Coffee and tea consumption and the prevalence of coronary heart disease in men and women. J. Epidemiol. Community Health. 47: 171-175

[6]. Bucolo G. and David H. (1973) Triglycerides enzymatic- spectrophotometric assay. Clin. Chem. 19: 476.

[7]. Burstein M., Scholnick H.R. and Morfin R. (1970) HDL cholesterol precipitating reagent. Phosphotungstate/ Magnesium. J. Lipid Res. 11: 583.

[8]. Chabrole E. and Charnnat R. (1937) Press. Med. 96: 1713.

[9]. Chan P., Fong W., Cheung Y., Huang Y., Ho W. and Chen Zhen-Yu. (1999) Jasmine green tea epicatechins are hypolipidemic in hamsters (Mesocricetus auratus) fed a high fat diet. J. Nutr. 129: 1094-1101.

[10]. De Vos S. and De Schrijver R. (2003) Lipid metabolism, intestinal fermentation and mineral absorption in rats consuming black tea. Nutr. Res. 23: 527-537.

[11]. Fossati P. and Prencipe L. (1982) Triglycerides enzymatic spectrophotometric assay. Clin. Chem. $28: 2077$.

[12]. Friedwald W.T., Levy R.I. and Fredrickson D.S. (1972) Estimation of the concentration of low-density lipoprotein cholesterol in plasma without use of the prevarative ultracentrifuge. Clin. Chem. 18: 499.

[13]. Gordon T., Castelli W. P. and Dawber T. R. (1977) High density lipoprotein as a protective factor against coronary heart dise ase. Am. J. Med. 62: 707-714.

[14]. Green M.S. and Harari G. (1992) Association of serum lipoproteins and health-related habits with coffee and tea consumption in free-living subjects examined in the Israeli CORDIS study. Prev. Med. 21: 532-545

[15]. Grove T.H. (1979) HDL cholesterol precipitating reagent. Phosphotungstate/ Magnesium. Clin. Chem. $25: 560$.

[16]. Hara Y., Luo J., Wickremashinghe L. and Yamanishi T. (1995) Chemical composition of tea. Food rev. Intern. 11: 435-525.

[17]. Hasegawa R., Chujo T., Sai-Kato K., Umemura T., Tanimura A. and KuroKawa Y. (1995) Preventive effects of green tea against DNA damage and hepatotoxicity in rats treated with 2-Nitropropane. Fd. Chem. Toxic. 33 (11): 961-970.

[18]. He P., Noda Y. and Sugiyama K. (2001) Green tea suppresses lipopolysaccharide-induced injury in D-Galactosamine-sensitized rats. J. Nutr. 131: 1560-1567.

[19]. Hikino H., Kiso Y., Hatano T., Yoshida T. and Okuda T. (1985) Antihepatotoxic actions of tannins. J. Ethnopharmacol. 14: 19-29.

[20]. Hollman P.C.H., Feskens E.J.M. and Katan M.B. (1999) Absorption, metabolism and bioavailability of flavonoids. In: Rice-Evans C. \& Packer L. Ed. Flavonoids in health and disease. (New York) : 483-522.

[21]. Imai K. and Nakachi K. (1995) Cross sectional study of drinking green tea on cardiovascular and liver diseases. B. M. J. 310: 693Imai 696.

[22]. Klatsky A.L., Petitti D.B., Armstrong M.A. and Friedman G.D. (1985) Coffee, tea and cholesterol. Am. J. Cardiol. 55: 577-578.

[23]. Kono S., Shinchi K., Ikeda N., Yanai F. and Imanishi K. (1992) Green tea consumption and serum lipid profiles: a cross-sectional study in Northern Kyushu, Japan. Prev. Med. 21: 526-531.

[24]. Li R. W., Douglas T. D., Maiyoh G. K., Adeli K. and Theriault A. G. (2006) The herbal supplement, Green tea leaf extract, Improves lipid and glucose homeostasis in a fructose-fed insulin- resistant hamster model. J. Ethnopharmacol. 104 (1-2): 24-31.

[25]. Lord V. (1999).www.consciouschoice.com

[26]. Miyagawa C., Wu C. and Kennedy D.O. (1997) Protective effect of green tea extract and tea polyphenols against the cytotoxicity of 1, 4-naphthoquinone in isolated rat hepatocytes. Bio. Sci. Biotechnol. Biochem. 61: 1901-1905.

[27]. Princen H.M., Van Duyvenvoorde W. and Buytenhek R. (1998) No effect of consumption of green and black tea on plasma lipid and antioxidant levels and on LDL oxidation in smokers. Arterioscler. Thromb. Vasc. Biol. 18: 833-841.

[28]. Ramesha G.S., Paul R. and Ganguly J. (1980) Effect of dietary unsaturated oils on the biosynthesis of cholesterol, and on biliary and fecal excretion of cholesterol and bile acid in rat. J. Nutr. 110: 2149-2158.

[29]. Richmond W. (1973) Preparation and properties of a cholesterol oxidase from Norcadia sp. and its application to the enzymatic assay of total cholesterol in serum. Clin. Chem. 19: 1350-1356.

[30]. Riemersma R.A., Rice-Evans C.A., Tyrrell R.M., Clifford M.N. and Lean M.E.J. (2001) Tea flavonoids and cardiovascular health. Q. J. Med. 94: 277-282.

[31]. Rimm E.B., Katan M.B. and Ascherio A. (1996) Relation between intake of flavonoids and risk for coronary heart disease in male health professionals. Ann. Intern. Med. 125: 384-389.

[32]. Rosenberg L., Palmer J.R. and Kelly J.P. (1988) Coffee drinking and nonfatal myocardial infarction in men under 55 years of age. Am. J. Epidemiol. 128: 570-578.

[33]. Sánchez E., Morales C R, Castillo S, Leos-Rivas C, García-Becerra L, and Martínez D MO (2016) “Antibacterial and antibiofilm activity of methanolic plant extracts against nosocomial microorganisms. Evidence-Based Complementary and Alternative Medicine, Article ID 1572697, 8 pages,. doi:10.1155/2016/157269 
[34]. Sanchez-Muniz F.J., Higon E., Cava F. and Viejo J.M. (1991) Acceptability of diets containing olive oil fried sardines (Sardina pilchardus) in the prevention of dietary hypercholesterolemia in rats. J. Sci. Food. Agric. 56: 155-156.

[35]. Shimizu M., Wada S., Hayashi T., Arisawa M., Ikegaya K., Ogaku S., Yano S. and Morita N. (1988) Studies on hypoglycemic constituents of Japanese tea. Yakugaku Zasshi (in Japanese). 108: 964-970.

[36]. Sugiyama K., He P., Wada S. and Saeki S. (1999) Teas and other beverages suppress D-galactosamine-induced liver injury in rats. J. Nutr. 129: 1361-1367.

[37]. Taherpour A., Hashemi A,, Erfanimanesh S., Taki E. (2016). Efficacy of methanolic extract of green and black teas against extended-spectrum $\beta$-Lactamase-producing Pseudomonas earuginosa. Pak J Pharm Sci. 29(4):1257-61

[38]. Teuscher A. and Richterich P. (1971) Schweiz. Med. Wochensohr. 101: 342-390.

[39]. Tietz N. (1987) The aminotransferases. In: W.B.Saunders Company. Fundamentals of clinical chemistry. $3^{\text {rd }}$ Ed. (Philadelphia). pp.369 \& pp.749.

[40]. Trinder P. (1969) Determination of blood glucose using 4- Aminophenazone. J. Clin. Path. 22: 246.

[41]. Troup R., Hayes J. H., Raatz S. K. , Thyagarajan B., Khaliq W., Jacobs D. R., Key N. S. Morawski, B. M., Kaiser D, Bank A. J. and Gross M. (2015) Effect of black tea intake on blood cholesterol concentrations in individuals with mild hypercholesterolemia: a diet-controlled randomized trial. J Acad Nutr Diet.; 115(2): 264-271.

[42]. Vinson J.A. (1998) Effect of green and black tea supplementation on lipids, lipid oxidation and fibrinogen in hamster: mechanisms for the epidemiological benefits of tea drinking. FEBS Lett. 433: 44-46.

[43]. Vinson J.A. and Zhang J. (2005) Black and green teas equally inhibit diabetic cataracts in a streptozotocin-induced rat model of diabetes. J. Agric. Food Chem. 53(9): 3710-3713.

[44]. Wada S., He P., Watanabe N., Sakata K. and Sugiyama K. (1999) Suppression of D-galactosamine-iduced rat liver injury by glycosidic flavonoids-rich fraction from green tea. Biosci. Biotechnol. Biochem. 63: 570-572.

[45]. Yang C. and Landau J. (2000) Effects of tea consumption on nutrition and health. J. Nutr. 130: 2409-2412.

[46]. Yang T. and Koo M. (2000) Chinese green tea lowers cholesterol level through an increase in fecal lipid excretion. Life Sci. 66(5): 411-423.

[47]. Zeyuan D., Bingying T., Xiaolin L., Jinming H. and Yifeng C. (1998) Effect of green tea and black tea on the blood glucose, the blood triglycerides, and antioxidation in aged rats. J. Agric. Food Chem. 46: 875-878.

Table 1: Effect of green tea and black tea consumption on serum triglycerides, total lipids and glucose levels in untreated and galactosamine treated rats.

\begin{tabular}{|c|c|c|c|c|}
\hline & Groups & Untreated rats & Treated rats & Significance \\
\hline \multirow{3}{*}{ 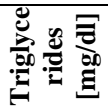 } & $\mathrm{C}$ & $98.88 \pm 36.39$ & $70.00 \pm 21.83$ & $*$ \\
\hline & GT & $83.19 \pm 27.25$ & $52.66 \pm 16.46$ & $*$ \\
\hline & BT & $98.09 \pm 14.02$ & $71.66 \pm 9.74$ & $*$ \\
\hline \multirow{3}{*}{ 吾昜 } & $\mathbf{C}$ & $2.45 \pm 0.53$ & $2.40 \pm 0.26$ & NS \\
\hline & GT & $2.36 \pm 0.36$ & $2.28 \pm 0.49$ & NS \\
\hline & BT & $1.06 \pm 0.31$ & $2.30 \pm 0.71$ & $*$ \\
\hline \multirow{3}{*}{ 苍 } & $\mathrm{C}$ & $140.21 \pm 25.05$ & $165.3 \pm 8.48$ & $*$ \\
\hline & GT & $122.98 \pm 11.57$ & $139.23 \pm 21.19$ & NS \\
\hline & BT & $113.45 \pm 18.38$ & $115.17 \pm 28.23$ & NS \\
\hline
\end{tabular}

C: Rats drinking water; GT: Rats drinking green tea; BT: Rats drinking black tea, NS: Non-significant; *: significant $(\mathrm{p}<0.05$; independent samples t-test)

Table 2: Effect of green tea and black tea consumption on serum total cholesterol, high density lipoproteinscholesterol and low density lipoproteins-cholesterol levels in untreated and galactosamine treated rats.

\begin{tabular}{|c|c|c|c|c|}
\hline & Groups & Untreated rats & Treated rats & Significance \\
\hline \multirow{3}{*}{ 家㿣 } & $\mathbf{C}$ & $55.11 \pm 20.08$ & $52.06 \pm 8.48$ & NS \\
\hline & GT & $51.93 \pm 11.14$ & $52.38 \pm 5.19$ & NS \\
\hline & BT & $35.18 \pm 10.29$ & $51.09 \pm 14.86$ & $*$ \\
\hline \multirow{3}{*}{ 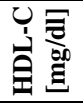 } & $\mathbf{C}$ & $13.40 \pm 2.52$ & $12.00 \pm 3.47$ & NS \\
\hline & GT & $10.91 \pm 3.10$ & $13.25 \pm 3.30$ & NS \\
\hline & BT & $12.35 \pm 3.41$ & $13.91 \pm 2.80$ & NS \\
\hline \multirow{3}{*}{ 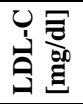 } & $\mathbf{C}$ & $28.22 \pm 18.54$ & $25.74 \pm 10.38$ & NS \\
\hline & GT & $29.75 \pm 11.41$ & $32.35 \pm 6.47$ & NS \\
\hline & BT & $11.06 \pm 6.10$ & $24.70 \pm 13.99$ & NS \\
\hline
\end{tabular}

NS: Non-significant; *: significant ( $\mathrm{p}<0.05$; independent samples t-test), CHOL: Total cholesterol; HDL-C: High density lipoproteins-cholesterol; LDL-C: Low density lipoproteins-cholesterol, C: Rats drinking water; GT: Rats drinking green tea; BT: Rats drinking black tea.

Table 3: Effect of green tea and black tea consumption on liver weight and hepatosomatic index in untreated and galactosamine treated rats

\begin{tabular}{|c|c|c|c|c|}
\hline & Groups & Untreated rats & Treated rats & Significance \\
\hline \multirow{3}{*}{ 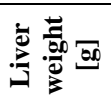 } & $\mathrm{C}$ & $11.17 \pm 1.08$ & $10.52 \pm 1.18$ & $\mathrm{NS}$ \\
\hline & GT & $10.11 \pm 1.25$ & $10.22 \pm 1.49$ & NS \\
\hline & BT & $10.55 \pm 1.30$ & $10.15 \pm 0.91$ & NS \\
\hline \multirow{3}{*}{ 鰙 } & $\mathbf{C}$ & $4.20 \pm 0.28$ & $4.08 \pm 0.30$ & NS \\
\hline & GT & $4.30 \pm 0.46$ & $4.23 \pm 0.40$ & NS \\
\hline & BT & $4.34 \pm 0.36$ & $4.27 \pm 0.27$ & NS \\
\hline
\end{tabular}

C: Rats drinking water; GT: Rats drinking green tea; BT: Rats drinking black tea, NS: Non-significant; *: significant $(\mathrm{p}<0.05$; independent samples t-test). 

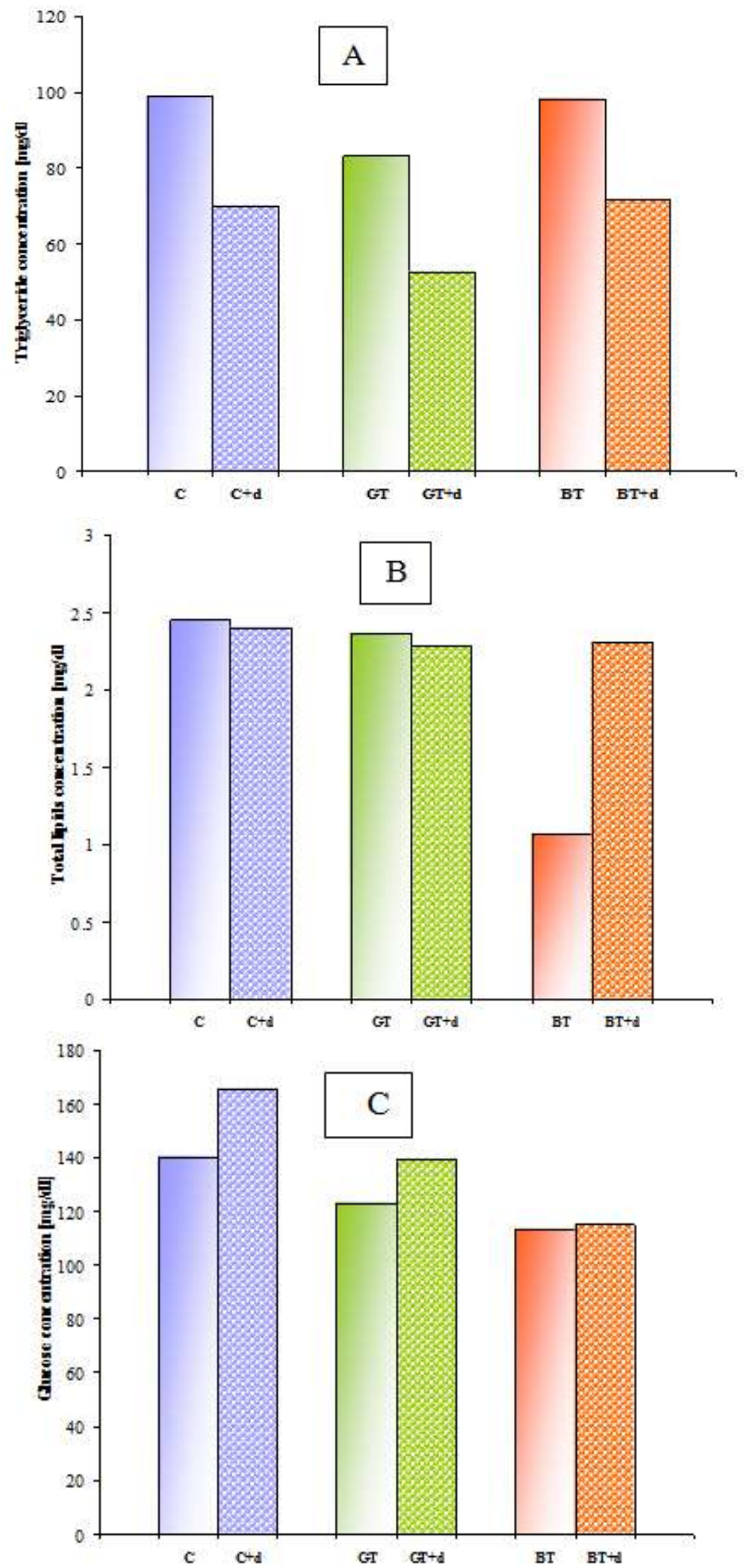

Figure1: Effect of green tea and black tea consumption on serum triglycerides levels (A), serum total lipids levels (B) and serum glucose levels (C) in untreated and galactosamine treated rats.

C: Rats drinking water; GT: Rats drinking green tea; BT: Rats drinking black tea, $\mathrm{C}+\mathrm{d}$ : Treated rats drinking water; $\mathrm{GT}+\mathrm{d}$ : Treated rats drinking green tea; $\mathrm{BT}+\mathrm{d}$ : Treated rats drinking black tea. 

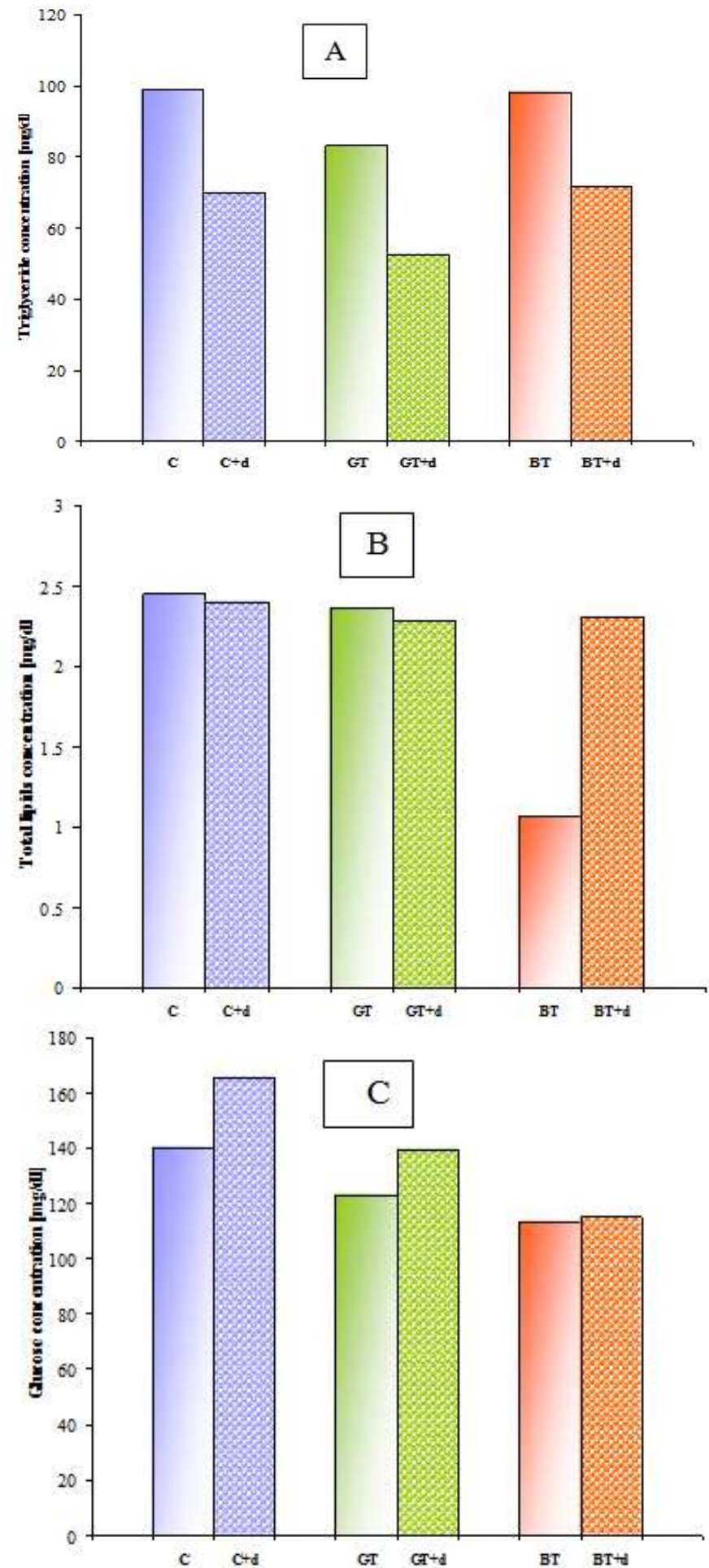

Figure-2. Effect of green tea and black tea consumption on serum total cholesterol levels (A), high density lipoproteins-cholesterol levels (B) and serum low density lipoproteins-cholesterol levels (C) in untreated and galactosamine treated rats.

C: Rats drinking water; GT: Rats drinking green tea; BT: Rats drinking black tea, C+ d: Treated rats drinking water; GT+ d: Treated rats drinking green tea; $\mathrm{BT}+\mathrm{d}$ : Treated rats drinking black tea. 

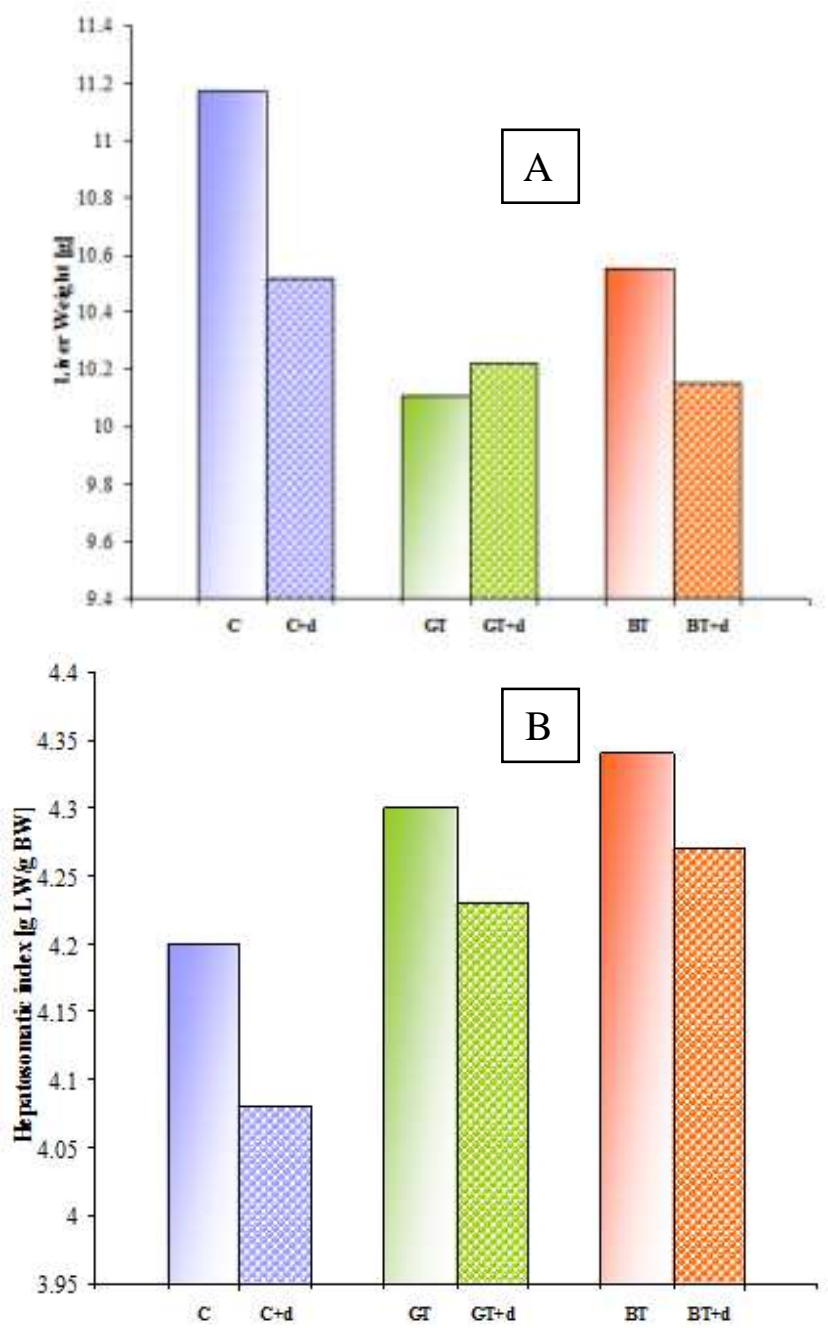

Figure-3. Effect of green tea and black tea consumption on liver weight (A) and hepatosomatic index (B) in untreated and galactosamine treated rats.

C: Rats drinking water; GT: Rats drinking green tea; BT: Rats drinking black tea, $\mathrm{C}+\mathrm{d}$ : Treated rats drinking water; $\mathrm{GT}+\mathrm{d}$ : Treated rats drinking green tea; $\mathrm{BT}+\mathrm{d}$ : Treated rats drinking black tea. 